\title{
Measurement of Physiological Fitness Level Among Male College Students in Dammam, Saudi Arabia Using the European Fitness Test-EuroFit
}

\author{
Hassan Soror Aboelwafa ${ }^{1}$, Ahmed Mahmoud Elkilany ${ }^{1} \&$ Mohammed Taha Al-Hariri ${ }^{2}$ \\ ${ }^{1}$ Self-Development Department, Preparatory Year Deanship and Supporting Studies, Imam Abdulrahman Bin \\ Faisal University, Dammam, KSA \\ ${ }^{2}$ Department of Physiology, College of Medicine, Imam Abdulrahman Bin Faisal University, Dammam, KSA \\ Correspondence: Mohammed Taha Al-Hariri, Department of Physiology, College of Medicine, Imam \\ Abdulrahman Bin Faisal University, P.O 2114-31451, Dammam, KSA. Tel: 966-50-727-5028. E-mail: \\ mtalhariri@iau.edu.sa
}

Received: July 17, 2019 Accepted: August 26, 2019 Online Published: September 2, 2019

doi:10.5539/gjhs.v11n11p21 URL: https://doi.org/10.5539/gjhs.v11n11p21

\begin{abstract}
Purpose: The purpose of this study was to measure the physiological fitness level of University students Saudi, male, using the European Fitness Test EuroFit.
\end{abstract}

Methods: The study was conducted on 451 Saudi, male, between 18-22 years age students. Physiological fitness was assessed using the eight tests included in the EuroFit battery. Body mass index (BMI) was assessed.

Results: The present study showed that, the majority of the participants $(55.7 \%)$ being within the normal body weight range and there was no significant difference between BMI categories in all components of fitness tests. According to the characteristics of the research sample, development of statistical tables of measurements derived from the process of conducting the EuroFit Fitness tests.

Conclusion: To the best of researchers' knowledge, it is the first study evaluated the physiological fitness and investigating the reliability of the Eurofit tests in the Gulf region. The results showed the determination of the students' fitness.

Keywords: student, fitness, weight, EuroFit

\section{Introduction}

In spite of the health benefits of the Physiological fitness, adolescent performance tests have decreased over the last three decades (Armstrong, 2012).

Physiological fitness status has been related to many health related outcomes. Inappropriate Fitness exercise in adolescence has been identified as a predictor for many health disorders such as, reduced quality of life and poor mental health. Cardiovascular diseases skeletal health (Ortega et al. 2012; Ruiz et al. 2009).

The World Health Organization [WHO] emphasized through many recommendations, the significance of physical activity for adults to gain many benefits, including improving cardiovascular fitness and musculoskeletal fitness, and in prevention of diseases (WHO, 2004).

The reference values are necessary to monitor the fitness status and categories the population. Nowadays, fitness status reference values in youth from different nations have been published (Berisha \& Cilli 2017).

The EuroFit tests are common and well-standardised tests offer a good opportunity to establish reference values. EuroFit test is used to assess the physiological fitness of children and youth according to Physics and Sports Council (Table 1) (Adam et al., 1988). Moreover, this test has been used widely to evaluate health-related physiological fitness for healthy and patients (Yurdalan, Kondu, \& Malkoç, 2007; Vancampfort et al., 2015).

The sports culture in the Middle East societies significantly lacks the importance of physiological fitness and practicing physical activity specially among youth of 18-19 years of age and this rate increased substantially with age (Mabry et al., 2010). 
There is also no scientific date for the levels of physiological fitness particularly the age groups of adults and young Arabic people.

Therefore, the aims of this study were to measure the physiological fitness level using the European Fitness Test EuroFit and provide specific fitness reference for Saudi Youth of Age group of 18-22 Years.

\section{Method}

\subsection{Participants and Sampling Procedures}

A cross sectional study was applied to 451 Saudi males aged 18-22 years, University students in the gymnasium of Imam Abdulrahman Bin Faisal University in Saudi Arabia.

Prior to participate in the study, an informed consent and a document with frequently asked questions were read by the participants. A written consent was obtained from all participants of the study. Measurements were carried out between April and Nov 2016-Feb 2017. The study was approved by the local committee and performed in accordance with the Helsinki Declaration (IRB No. 2014-14-221).

\subsection{Measures}

The tools needed for each test were prepared and their readiness was ensured to perform the tests on them. Each test was carried out under the supervision of $\mathrm{PhD}$ holder researchers and by the assistants of science degrees in physical activity. Educational films, including detailed testing procedures, instructions for each test and the tools needed for some tests were presented.

BMI was calculated as body mass in kilograms divided by the square of stature in meters $(\mathrm{kg} / \mathrm{m} 2)$ (Al-Hariri, Elkilany, \& Alkahtani, 2018). We have used BMI categories suggested by The National Institutes of Health and the World Health Organization (Alkahtani, Elkilany, \& Alhariri, 2015).

BMI Categories:

Underweight $=<18.5$

Normal weight $=18.5-24.9$

Overweight $=25-29.9$

Obesity $=$ BMI of 30 or greater

Exclusion criteria were problems of the lower extremities, dizziness, and other neurological symptoms that might be aggravated by the tests.

Physiological fitness was assessed using the eight tests included in the EuroFit battery (ERIKOĞLU et al. 2015): flamingo balance test, plate tapping test, flexibility test, standing broad jump test, handgrip test, sit-ups in 30 seconds test, bent arm hang test and 10x5-meter shuttle run test.

Before applying each test of EuroFit, the performance method was explained to the participants and they were given an appropriate opportunity to warm up before each test except the flexibility test.

A pilot study was conducted initially to measure the European Fitness Tests for 30 students to find out the coefficients (reliability, validity) of testing, using the test-retest method. There was a five day interval between the measurements of the same group of students.

\subsection{Data Analysis}

Statistical analysis was performed using IBM SPSS 19. Raw Data of the measurements extracted from the process were recorded in registration statements for each test. An alpha level of $p \leq 0.05$ was used as the criterion to determine significance for the reliability. Data was presented as mean and standard deviation of the mean. Fitness levels by BMI differences were tested by using ANOVA, $\mathrm{T}$ test and the significance level was set at $\mathrm{p} \leq 0.05$.

\section{Results}

\subsection{Sample Characteristics}

The total of included participants was 451Saudi youth male. The average age and BMI were $18.9 \pm 0.05$ and $24.6 \pm 0.03$ respectively as shown in Table 2 . Of the 451 participants, $12(2.7 \%)$ were underweight (UW), 251 $(55.7 \%)$ were normal weight $(\mathrm{NW}), 164(36.4 \%)$ were overweight $(\mathrm{OW})$, and $24(5.3 \%)$ were Obese $(\mathrm{O})$ as shown in Figure 1.

\subsection{Validation of the Pilot Study}

Table 3 shows that there is a positive significant relationship between the first and second pilot study's 
measurements. The correlation coefficients ranged from 0.800 to 0.970 which indicates that the reliability coefficients are very high. Moreover, the self-validity of the used tests in the study is also accepted. Mean \pm Standard deviations (SD) for all studied testes were shown in Table 4. The statistical tables presented below (Table 5 - Table 12), showed the raw scores extracted from the measurement process, Z Score and T Score for each test of the EuroFit Fitness Test Battery.

\subsection{Flamingo Balance Test}

Table 5 indicates that the best level achieved during the Flamingo Balance Test was 4.00 times. It is the number of falling times during balance on the bar for 1.00 minutes. The lowest level achieved was 23 times.

\subsection{Plate Tapping Test}

Table 6 indicates that the best level achieved during the Plate Tapping Test was 8.00 seconds. The lowest time achieved by tapping on each plate was 25 times. The lowest level achieved was 25.74 seconds.

\subsection{Flexibility Test}

Table 7 indicates that the best level achieved during the Flexibility Test was $27.00 \mathrm{~cm}$. It is the largest distance by which the tests could achieve on the wall bar. The lowest level achieved was $20.00-\mathrm{cm}$.

\subsection{Standing Broad Jump Test}

Table 8 indicates that the best level achieved during the Standing Broad Jump Test was $250.00 \mathrm{~cm}$. It is the largest distance the tests could achieve during the horizontal jump on the ground. The lowest level achieved was 90.00 $\mathrm{cm}$.

\subsection{Handgrip Test}

Table 9 indicates that the best level achieved during the Handgrip Test was $59.40 \mathrm{~kg}$. It is the largest strength recorded on the handgrip device during the test. The lowest level achieved was $41.60 \mathrm{~kg}$.

\subsection{Sit-Ups Test}

Table 10 indicates that the best level achieved during the Sit-Ups in 30 Seconds Test was 31.00 times. It is the greatest number of times achieved during the performance of the Sit-Ups in 30 Seconds Test. The lowest level achieved was 2.00 times.

\subsection{Bent Arm Hang Test}

Table 11 indicates that the best level achieved during the Bent Arm Hang Test was 71.10 seconds. It is the largest period achieved by arm hanging on the bar. The lowest level achieved was 0.00 second. This means that the tests did not achieve any time during hanging, that is, failure to hang on the bar.

\subsection{Meter Shuttle Run Test}

Table 12 indicates that the best level achieved during the 10X5-Meter Shuttle Run Test was 13.30 seconds. It is the lowest period time achieved during shuttle run. The lowest level achieved was 36.20 seconds.

Our study didn't show any significant differences of the used tests between the different weight category groups.
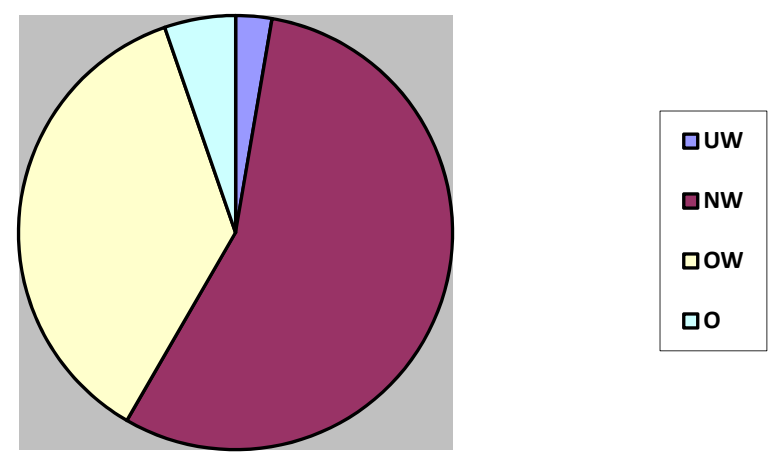

Figure 1. Body Mass categories of the participants

UN: underweight; NW: normal weight; OW: overweight; O: Obese 
Table 1. EuroFit Test Protocol (Council of Europe, 1987)

\begin{tabular}{lll}
\hline Hand grip & Strength & $\begin{array}{l}\text { Static strength } \\
\text { Explosive strength }\end{array}$ \\
Standing broad jump & & Functional strength \\
Bent arm hang & Muscular endurance & Trunk strength \\
Sit-ups & & Running speed - agility \\
\hline Shuttle run: $10 \times 5$ meters & Speed & Speed of limb movement \\
Plate tapping & & Flexibility \\
\hline Sit and reach & Flexibility & Total body balance \\
\hline Flamingo balance & Balance &
\end{tabular}

Table 2. Characteristics of research sample

\begin{tabular}{ll}
\hline & Mean $\pm S D$ \\
\hline Age & $18.9 \pm 0.05$ \\
$B M I$ & $24.6 \pm 0.03$ \\
\hline
\end{tabular}

Table 3. The coefficients (Reliability, Validity) of the European Fitness Test -EuroFit

\begin{tabular}{lllllll}
\hline \multirow{2}{*}{ Tests } & Testing & \multicolumn{3}{l}{ Retesting } & Reliability Coefficient & Validity Coefficient \\
\cline { 2 - 5 } & SD & M & SD & M & & 0.950 \\
\hline Flamingo Balance Test & 14.833 & 5.052 & 14.800 & 4.390 & 0.900 & 0.890 \\
Plate Tapping Test & 15.267 & 2.160 & 15.294 & 2.069 & 0.800 & 0.980 \\
Flexibility Test & 3.917 & 8.264 & 3.567 & 8.292 & 0.960 & 0.890 \\
Standing Broad Jump & 174.233 & 25.998 & 176.900 & 24.433 & 0.970 & 0.980 \\
Handgrip Test & 37.259 & 7.697 & 37.748 & 8.009 & 0.960 & 0.950 \\
Sit-Ups In 30 Seconds Test & 22.300 & 3.261 & 22.100 & 3.428 & 0.900 & 0.930 \\
Bent Arm Hang Test & 17.036 & 16.408 & 17.088 & 16.993 & 0.860 & 0.930 \\
10X5-Meter Shuttle Run Test & 20.677 & 2.983 & 21.333 & 2.834 & 0.860 & \\
\hline
\end{tabular}

Table 4. Mean \pm SD of the European Fitness Tests -EuroFit

\begin{tabular}{lll}
\hline \multirow{2}{*}{ Tests } & \multicolumn{2}{l}{ Testing } \\
\cline { 2 - 3 } & Mean & SD \\
\hline Flamingo Balance Test & 15.1 & 5.1 \\
Plate Tapping Test & 19.1 & 6.5 \\
Flexibility Test & 7 & 5 \\
Standing Broad Jump & 1.7 & 9.7 \\
Handgrip Test & 36.7 & 7.3 \\
Sit-Ups In 30 Seconds Test & 17.5 & 5.0 \\
Bent Arm Hang Test & 14.2 & 1.5 \\
10X5-Meter Shuttle Run Test & 22.8 & 3.3 \\
\hline
\end{tabular}

SD: slandered deviation. 
Table 5. Raw Data, Z Score and T Score of Flamingo Balance Test

\begin{tabular}{llllll}
\hline Raw Data & Z Score & T Score & Raw Data & Z Score & T Score \\
\hline 4.00 & $1.83-$ & 31.70 & 14.00 & 0.10 & 50.96 \\
5.00 & $1.64-$ & 33.62 & 15.00 & 0.29 & 52.89 \\
6.00 & $1.45-$ & 35.55 & 16.00 & 0.48 & 54.82 \\
7.00 & $1.25-$ & 37.48 & 17.00 & 0.67 & 56.74 \\
8.00 & $1.06-$ & 39.40 & 18.00 & 0.87 & 58.67 \\
9.00 & $0.87-$ & 41.33 & 19.00 & 1.06 & 60.60 \\
10.00 & $0.67-$ & 43.26 & 20.00 & 1.25 & 62.52 \\
11.00 & $0.48-$ & 45.18 & 21.00 & 1.45 & 64.45 \\
12.00 & $0.29-$ & 47.11 & 22.00 & 1.64 & 66.38 \\
13.00 & $0.10-$ & 49.04 & 23.00 & 1.83 & 68.30 \\
\hline
\end{tabular}

Table 6. Raw Data, Z Score and T Score of Plate Tapping Test

\begin{tabular}{llllll}
\hline Raw Data & Z Score & T Score & Raw Data & Z Score & T Score \\
\hline 8.00 & $2.48-$ & 25.16 & 18.43 & 0.14 & 51.44 \\
10.50 & $1.85-$ & 31.46 & 18.62 & 0.19 & 51.91 \\
11.10 & $1.70-$ & 32.97 & 19.00 & 0.29 & 52.87 \\
11.94 & $1.49-$ & 35.09 & 19.01 & 0.29 & 52.90 \\
12.56 & $1.34-$ & 36.65 & 19.03 & 0.29 & 52.95 \\
12.76 & $1.28-$ & 37.15 & 19.80 & 0.49 & 54.89 \\
13.00 & $1.22-$ & 37.76 & 19.87 & 0.51 & 55.06 \\
13.07 & $1.21-$ & 37.93 & 19.94 & 0.52 & 55.24 \\
13.70 & $1.05-$ & 39.52 & 20.00 & 0.54 & 55.39 \\
14.00 & $0.97-$ & 40.28 & 20.01 & 0.54 & 55.42 \\
14.30 & $0.90-$ & 41.03 & 20.22 & 0.59 & 55.94 \\
14.91 & $0.74-$ & 42.57 & 20.50 & 0.66 & 56.65 \\
15.00 & $0.72-$ & 42.80 & 21.00 & 0.79 & 57.91 \\
15.20 & $0.67-$ & 43.30 & 21.07 & 0.81 & 58.09 \\
15.95 & $0.48-$ & 45.19 & 21.09 & 0.81 & 58.14 \\
16.00 & $0.47-$ & 45.31 & 21.43 & 0.90 & 58.99 \\
16.20 & $0.42-$ & 45.82 & 21.73 & 0.97 & 59.75 \\
17.26 & $0.15-$ & 48.49 & 22.00 & 1.04 & 60.43 \\
17.30 & $0.14-$ & 48.59 & 22.45 & 1.16 & 61.56 \\
17.32 & $0.14-$ & 48.64 & 22.60 & 1.19 & 61.94 \\
17.80 & $0.02-$ & 49.85 & 23.00 & 1.29 & 62.95 \\
18.00 & 0.04 & 50.35 & 23.50 & 1.42 & 64.21 \\
18.01 & 0.04 & 50.38 & 25.00 & 1.80 & 67.98 \\
18.40 & 0.14 & 51.36 & 25.74 & 1.98 & 69.85 \\
\hline & & & & &
\end{tabular}


Table 7. Raw Data, Z Score and T Score of Flexibility Test

\begin{tabular}{|c|c|c|c|c|c|}
\hline Raw Data & Z Score & T Score & Raw Data & Z Score & T Score \\
\hline $20-$ & 1.79 & 32.13 & 2 & 0.02 & 50.21 \\
\hline $19-$ & $1.71-$ & 32.95 & 3 & 0.1 & 51.03 \\
\hline $17-$ & $1.54-$ & 34.59 & 3.5 & 0.14 & 51.44 \\
\hline $16-$ & $1.46-$ & 35.41 & 4 & 0.18 & 51.85 \\
\hline $15-$ & $1.38-$ & 36.24 & 5 & 0.27 & 52.67 \\
\hline $14.5-$ & $1.34-$ & 36.65 & 5.5 & 0.31 & 53.08 \\
\hline $14-$ & 1.29 & 37.06 & 6 & 0.35 & 53.49 \\
\hline $13.5-$ & $1.25-$ & 37.47 & 6.5 & 0.39 & 53.9 \\
\hline $13-$ & $1.21-$ & 37.88 & 7 & 0.43 & 54.31 \\
\hline $12.5-$ & $1.17-$ & 38.29 & 7.5 & 0.47 & 54.72 \\
\hline $12-$ & $1.13-$ & 38.7 & 8 & 0.51 & 55.14 \\
\hline $11-$ & $1.05-$ & 39.52 & 8.5 & 0.55 & 55.55 \\
\hline $10-$ & $0.97-$ & 40.35 & 9 & 0.6 & 55.96 \\
\hline 9.5- & $0.92-$ & 40.76 & 10 & 0.68 & 56.78 \\
\hline 9- & $0.88-$ & 41.17 & 11 & 0.76 & 57.6 \\
\hline $8.5-$ & $0.84-$ & 41.58 & 12 & 0.84 & 58.42 \\
\hline 8- & $0.8-$ & 41.99 & 12.5 & 0.88 & 58.83 \\
\hline $7-$ & $0.72-$ & 42.81 & 13 & 0.92 & 59.24 \\
\hline $6-$ & $0.64-$ & 43.63 & 14 & 1.01 & 60.07 \\
\hline $5.5-$ & $0.6-$ & 44.04 & 14.5 & 1.05 & 60.48 \\
\hline $5.25-$ & $0.58-$ & 44.25 & 15 & 1.09 & 60.89 \\
\hline $5-$ & $0.55-$ & 44.45 & 16 & 1.17 & 61.71 \\
\hline 4.5- & $0.51-$ & 44.86 & 16.32 & 1.2 & 61.97 \\
\hline $4-$ & $0.47-$ & 45.28 & 17 & 1.25 & 62.53 \\
\hline $3-$ & $0.39-$ & 46.1 & 17.5 & 1.29 & 62.94 \\
\hline $2.5-$ & $0.35-$ & 46.51 & 18 & 1.34 & 63.35 \\
\hline $2-$ & $0.31-$ & 46.92 & 19 & 1.42 & 64.17 \\
\hline $1.5-$ & $0.27-$ & 47.33 & 20 & 1.5 & 65 \\
\hline $1-$ & $0.23-$ & 47.74 & 23 & 1.75 & 67.46 \\
\hline 0 & $0.14-$ & 48.56 & 26 & 1.99 & 69.93 \\
\hline 1 & $0.06-$ & 49.38 & 27 & 2.07 & 70.75 \\
\hline
\end{tabular}


Table 8. Raw Data, Z Score and T Score of Standing Broad Jump Test

\begin{tabular}{llllll}
\hline Raw Data & $\boldsymbol{Z}$ Score & T Score & Raw Data & Z Score & T Score \\
\hline 90 & $2.11-$ & 28.87 & 181 & 0.07 & 50.74 \\
100 & $1.87-$ & 31.27 & 182 & 0.1 & 50.98 \\
105 & $1.75-$ & 32.48 & 185 & 0.17 & 51.7 \\
110 & $1.63-$ & 33.68 & 186 & 0.19 & 51.94 \\
115 & $1.51-$ & 34.88 & 187 & 0.22 & 52.18 \\
120 & $1.39-$ & 36.08 & 190 & 0.29 & 52.9 \\
125 & $1.27-$ & 37.28 & 194 & 0.39 & 53.86 \\
130 & $1.15-$ & 38.48 & 195 & 0.41 & 54.1 \\
135 & $1.03-$ & 39.69 & 198 & 0.48 & 54.83 \\
138 & $0.96-$ & 40.41 & 200 & 0.53 & 55.31 \\
140 & $0.91-$ & 40.89 & 205 & 0.65 & 56.51 \\
145 & $0.79-$ & 42.09 & 206 & 0.67 & 56.75 \\
146 & $0.77-$ & 42.33 & 210 & 0.77 & 57.71 \\
147 & $0.74-$ & 42.57 & 211 & 0.8 & 57.95 \\
150 & $0.67-$ & 43.29 & 213 & 0.84 & 58.43 \\
155 & $0.55-$ & 44.49 & 215 & 0.89 & 58.91 \\
156 & $0.53-$ & 44.73 & 220 & 1.01 & 60.11 \\
158 & $0.48-$ & 45.21 & 221 & 1.04 & 60.35 \\
160 & $0.43-$ & 45.69 & 225 & 1.13 & 61.31 \\
165 & $0.31-$ & 46.89 & 230 & 1.25 & 62.52 \\
170 & $0.19-$ & 48.1 & 234 & 1.35 & 63.48 \\
175 & $0.07-$ & 49.3 & 235 & 1.37 & 63.72 \\
176 & $0.05-$ & 49.54 & 240 & 1.49 & 64.92 \\
177 & $0.02-$ & 49.78 & 245 & 1.61 & 66.12 \\
178 & 0 & 50.02 & 248 & 1.68 & 66.84 \\
180 & 50.5 & 250 & 1.73 & 67.32 \\
\hline & 0.05 & & & &
\end{tabular}

Table 9. Raw Data, Z Score and T Score of Handgrip Test

\begin{tabular}{llllll}
\hline Raw Data & Z Score & T Score & Raw Data & Z Score & T Score \\
\hline 17.90 & $1.90-$ & 31.00 & 38.10 & 0.10 & 51.02 \\
18.70 & $1.82-$ & 31.79 & 38.30 & 0.12 & 51.22 \\
20.40 & $1.65-$ & 33.48 & 38.50 & 0.14 & 51.42 \\
24.10 & $1.29-$ & 37.15 & 38.60 & 0.15 & 51.52 \\
24.40 & $1.26-$ & 37.44 & 39.60 & 0.25 & 52.51 \\
25.00 & $1.20-$ & 38.04 & 39.80 & 0.27 & 52.71 \\
25.07 & $1.19-$ & 38.11 & 39.90 & 0.28 & 52.80 \\
25.10 & $1.19-$ & 38.14 & 40.00 & 0.29 & 52.90 \\
26.70 & $1.03-$ & 39.72 & 42.43 & 0.53 & 55.31 \\
26.80 & $1.02-$ & 39.82 & 42.70 & 0.56 & 55.58 \\
26.90 & $1.01-$ & 39.92 & 42.87 & 0.57 & 55.75 \\
\hline
\end{tabular}




\begin{tabular}{|c|c|c|c|c|c|}
\hline 27.00 & $1.00-$ & 40.02 & 45.50 & 0.84 & 58.35 \\
\hline 27.09 & 0.99- & 40.11 & 45.80 & 0.87 & 58.65 \\
\hline 27.50 & $0.95-$ & 40.52 & 45.90 & 0.88 & 58.75 \\
\hline 28.20 & $0.88-$ & 41.21 & 46.00 & 0.89 & 58.85 \\
\hline 28.30 & $0.87-$ & 41.31 & 46.10 & 0.89 & 58.95 \\
\hline 29.90 & $0.71-$ & 42.89 & 46.40 & 0.92 & 59.25 \\
\hline 30.00 & $0.70-$ & 42.99 & 46.60 & 0.94 & 59.44 \\
\hline 30.01 & $0.70-$ & 43.00 & 46.70 & 0.95 & 59.54 \\
\hline 30.10 & $0.69-$ & 43.09 & 46.90 & 0.97 & 59.74 \\
\hline 31.60 & $0.54-$ & 44.58 & 47.00 & 0.98 & 59.84 \\
\hline 31.70 & $0.53-$ & 44.68 & 47.10 & 0.99 & 59.94 \\
\hline 31.90 & $0.51-$ & 44.88 & 50.60 & 1.34 & 63.41 \\
\hline 32.00 & $0.50-$ & 44.98 & 50.70 & 1.35 & 63.51 \\
\hline 32.01 & $0.50-$ & 44.99 & 50.90 & 1.37 & 63.71 \\
\hline 35.02 & $0.20-$ & 47.97 & 51.30 & 1.41 & 64.10 \\
\hline 35.71 & $0.13-$ & 48.65 & 52.80 & 1.56 & 65.59 \\
\hline 37.70 & 0.06 & 50.62 & 58.70 & 2.14 & 71.44 \\
\hline 37.80 & 0.07 & 50.72 & 59.20 & 2.19 & 71.93 \\
\hline 38.00 & 0.09 & 50.92 & & & \\
\hline
\end{tabular}

Table 10. Raw Data, Z Score and T Score of Sit-Ups In 30 Seconds Test

\begin{tabular}{llllll}
\hline Raw Data & Z Score & T Score & Raw Data & Z Score & T Score \\
\hline 2 & $1.7-$ & 32.96 & 18 & 0.13 & 51.31 \\
3 & $1.59-$ & 34.11 & 19 & 0.25 & 52.45 \\
4 & $1.47-$ & 35.25 & 20 & 0.36 & 53.6 \\
5 & $1.36-$ & 36.4 & 21 & 0.47 & 54.75 \\
7 & $1.13-$ & 38.69 & 22 & 0.59 & 55.89 \\
8 & $1.02-$ & 39.84 & 23 & 0.7 & 57.04 \\
9 & $0.9-$ & 40.99 & 24 & 0.82 & 58.19 \\
10 & $0.79-$ & 42.13 & 25 & 0.93 & 59.33 \\
11 & $0.67-$ & 43.28 & 26 & 1.05 & 60.48 \\
12 & $0.56-$ & 44.43 & 27 & 1.16 & 61.63 \\
13 & $0.44-$ & 45.57 & 28 & 1.28 & 62.78 \\
14 & $0.33-$ & 46.72 & 29 & 1.39 & 63.92 \\
15 & $0.21-$ & 47.87 & 30 & 1.51 & 65.07 \\
16 & $0.1-$ & 49.01 & 31 & 1.62 & 66.22 \\
17 & 0.02 & 50.16 & & & \\
\hline
\end{tabular}


Table 11. Raw Data, Z Score and T Score of Bent Arm Hang Test

\begin{tabular}{|c|c|c|c|c|c|}
\hline Raw Data & Z Score & T Score & Raw Data & Z Score & T Score \\
\hline .00 & $1.04-$ & 39.65 & 10.32 & $0.51-$ & 44.90 \\
\hline 1.00 & $0.98-$ & 40.16 & 11.90 & $0.43-$ & 45.71 \\
\hline 1.30 & $0.97-$ & 40.31 & 12.09 & $0.42-$ & 45.80 \\
\hline 1.73 & $0.95-$ & 40.53 & 12.17 & $0.42-$ & 45.85 \\
\hline 1.98 & $0.93-$ & 40.66 & 16.49 & $0.20-$ & 48.04 \\
\hline 2.00 & $0.93-$ & 40.67 & 16.50 & $0.20-$ & 48.05 \\
\hline 2.30 & $0.92-$ & 40.82 & 16.91 & $0.17-$ & 48.26 \\
\hline 2.45 & $0.91-$ & 40.90 & 17.25 & $0.16-$ & 48.43 \\
\hline 2.50 & $0.91-$ & 40.92 & 35.50 & 0.77 & 57.72 \\
\hline 2.51 & $0.91-$ & 40.93 & 35.70 & 0.78 & 57.83 \\
\hline 2.54 & $0.91-$ & 40.94 & 35.72 & 0.78 & 57.84 \\
\hline 2.60 & $0.90-$ & 40.97 & 36.00 & 0.80 & 57.98 \\
\hline 3.00 & $0.88-$ & 41.18 & 36.20 & 0.81 & 58.08 \\
\hline 3.13 & $0.88-$ & 41.24 & 36.26 & 0.81 & 58.11 \\
\hline 3.20 & $0.87-$ & 41.28 & 36.46 & 0.82 & 58.21 \\
\hline 3.28 & $0.87-$ & 41.32 & 36.60 & 0.83 & 58.28 \\
\hline 4.80 & $0.79-$ & 42.09 & 37.32 & 0.87 & 58.65 \\
\hline 4.85 & $0.79-$ & 42.12 & 37.80 & 0.89 & 58.90 \\
\hline 5.00 & $0.78-$ & 42.19 & 37.90 & 0.89 & 58.95 \\
\hline 5.11 & $0.77-$ & 42.25 & 38.00 & 0.90 & 59.00 \\
\hline 6.35 & $0.71-$ & 42.88 & 45.02 & 1.26 & 62.57 \\
\hline 6.71 & $0.69-$ & 43.07 & 45.05 & 1.26 & 62.59 \\
\hline 7.00 & $0.68-$ & 43.21 & 45.21 & 1.27 & 62.67 \\
\hline 7.17 & $0.67-$ & 43.30 & 48.66 & 1.44 & 64.42 \\
\hline 7.37 & $0.66-$ & 43.40 & 49.97 & 1.51 & 65.09 \\
\hline 7.50 & $0.65-$ & 43.47 & 50.45 & 1.53 & 65.34 \\
\hline 9.05 & $0.57-$ & 44.26 & 50.57 & 1.54 & 65.40 \\
\hline 9.20 & $0.57-$ & 44.33 & 60.00 & 2.02 & 70.20 \\
\hline 10.24 & $0.51-$ & 44.86 & 65.00 & 2.27 & 72.74 \\
\hline 10.28 & $0.51-$ & 44.88 & 71.10 & 2.59 & 75.85 \\
\hline
\end{tabular}


Table 12. Raw Data, Z Score and T Score of 10X5-Meter Shuttle Run Test

\begin{tabular}{|c|c|c|c|c|c|}
\hline Raw Data & Z Score & T Score & Raw Data & Z Score & T Score \\
\hline 13.30 & $1.85-$ & 31.48 & 22.15 & $0.11-$ & 48.93 \\
\hline 14.30 & $1.65-$ & 33.45 & 23.66 & 0.19 & 51.91 \\
\hline 14.56 & $1.60-$ & 33.96 & 23.70 & 0.20 & 51.99 \\
\hline 14.74 & $1.57-$ & 34.32 & 23.75 & 0.21 & 52.09 \\
\hline 14.80 & $1.56-$ & 34.44 & 23.80 & 0.22 & 52.19 \\
\hline 16.32 & $1.26-$ & 37.44 & 24.27 & 0.31 & 53.12 \\
\hline 16.40 & $1.24-$ & 37.59 & 24.37 & 0.33 & 53.31 \\
\hline 16.88 & $1.15-$ & 38.54 & 24.46 & 0.35 & 53.49 \\
\hline 17.14 & $1.09-$ & 39.05 & 24.47 & 0.35 & 53.51 \\
\hline 17.15 & $1.09-$ & 39.07 & 24.48 & 0.35 & 53.53 \\
\hline 18.40 & $0.85-$ & 41.54 & 24.51 & 0.36 & 53.59 \\
\hline 18.70 & $0.79-$ & 42.13 & 25.28 & 0.51 & 55.11 \\
\hline 18.76 & $0.78-$ & 42.25 & 25.30 & 0.51 & 55.15 \\
\hline 19.31 & $0.67-$ & 43.33 & 25.45 & 0.54 & 55.44 \\
\hline 19.32 & $0.66-$ & 43.35 & 25.48 & 0.55 & 55.50 \\
\hline 19.33 & $0.66-$ & 43.37 & 26.40 & 0.73 & 57.32 \\
\hline 19.34 & $0.66-$ & 43.39 & 26.41 & 0.73 & 57.34 \\
\hline 19.40 & $0.65-$ & 43.51 & 26.60 & 0.77 & 57.71 \\
\hline 19.44 & $0.64-$ & 43.59 & 26.63 & 0.78 & 57.77 \\
\hline 19.45 & $0.64-$ & 43.61 & 26.70 & 0.79 & 57.91 \\
\hline 20.08 & $0.51-$ & 44.85 & 27.27 & 0.90 & 59.03 \\
\hline 20.09 & $0.51-$ & 44.87 & 27.35 & 0.92 & 59.19 \\
\hline 20.10 & $0.51-$ & 44.89 & 27.37 & 0.92 & 59.23 \\
\hline 20.16 & $0.50-$ & 45.01 & 27.60 & 0.97 & 59.68 \\
\hline 21.69 & $0.20-$ & 48.03 & 28.30 & 1.11 & 61.07 \\
\hline 21.70 & $0.20-$ & 48.05 & 28.54 & 1.15 & 61.54 \\
\hline 21.74 & $0.19-$ & 48.13 & 28.83 & 1.21 & 62.11 \\
\hline 21.78 & $0.18-$ & 48.21 & 29.00 & 1.24 & 62.45 \\
\hline 21.80 & $0.18-$ & 48.24 & 32.38 & 1.91 & 69.11 \\
\hline 21.89 & $0.16-$ & 48.42 & 33.23 & 2.08 & 70.79 \\
\hline 21.93 & $0.15-$ & 48.50 & 33.56 & 2.14 & 71.44 \\
\hline 21.99 & $0.14-$ & 48.62 & 35.20 & 2.47 & 74.67 \\
\hline 22.00 & $0.14-$ & 48.64 & 36.20 & 2.66 & 76.65 \\
\hline 22.14 & $0.11-$ & 48.92 & & & \\
\hline
\end{tabular}

\section{Discussion}

To the best of researchers' knowledge, it is the first study evaluated the physiological fitness and investigating the reliability of the Eurofit tests in the Gulf region.

The present study provides a new score $(\mathrm{Z} \& \mathrm{~T})$ for different tests of fitness.

In this study, no significant difference was found between BMI categories in all components of fitness tests. Based on the reported data (ERIKOĞLU et al., 2015; Lovecchio et al., 2012) and through the experience of the authors in the field of physical education and academic student activities, there was a need for improved the sportive activity of young people during the stage. There was also a clear variation in the performance level of participants in the performance of the tests. 
The calculated $\mathrm{Z}$ score and $\mathrm{T}$ Score is a very important reference for comparing individuals with their peers during the same age group. Rationing the fitness tests by setting guiding standard levels that can be used to compare the individuals' performance with their peers during the age group. This can clarify the deficiencies and weaknesses in educational curricula. Therefore, they could consider as a guide and a manual for specialists and educational leaders.

At this age, youth's daily lives are characterized by sedentary, gathering information on the level of physical activity is highly needed to identify the extent of developmental change in the level of fitness and to evaluating their general performance level based on reference data. In addition, many health related problems such as Diabetes and obesity resulting from a lack of physical activity in this age group can have adverse health consequences later in life (Reilly \& Kelly, 2011).

Monitoring physical activity among youth based on a solid scientific data will improve the health outcomes specially in gulf region where their less physical activity and sedentary life style are important risk factors for several diseases such as type 2 diabetes, cardiovascular diseases, and colon cancer (Musaiger, 2012). Moreover, diets high in fats and carbohydrates is very important contributing factors for obesity in the Middle East (Washi \& Ageib, 2010; Ng et al., 2011).

\section{Conclusion}

Through the results of this research, the training load can be rationed for University student in the 18-22 years age group. It can also design sports programs that can be achieved by figuring out the levels of physiological fitness among young people to underline the deficiencies in physical education programs at universities. It also aims at avoiding excessive overload greater than individuals' abilities, which can affect the growth stages of this age group.

These standard tables should be reevaluated at varying intervals with the progress in individual levels to be updated.

\section{Limitation}

There is no previously reported date of similar work at the local and regional level to compare the strengths of the physical capabilities of the studied sample with.

\section{Declarations}

\section{Ethics Approval and Consent to Participate}

An ethics application, including method section, protocols, participant information and informed consent was submitted to and approved by the ethics committee of Imam Abdulrahman Bin Faisal University, KSA (IRB No. 2014-14-221). The study was conducted in accordance with the Declaration of Helsinki. Participants received written and oral information about the project before the consent form was signed.

\section{Consent for Publication}

Not applicable.

\section{Availability of Data and Materials}

The data sets used and/or analysed during the current study are available from the corresponding author on reasonable request.

\section{Funding}

No funding was available for this study.

\section{Authors' Contributions}

HA and AK designed and planned the project. HA collected data, performed data analysis, and drafted and completed the manuscript. MA participated in every part of the data analysis. HA, AK, and MA participated in analysis meetings and commented on the manuscript. All authors read and approved the final version to be published.

\section{Acknowledgments}

The authors would like to thank the participants of this study.

\section{Competing Interests Statements}

The authors declare that they have no competing interests. No external funding was received for this work. 


\section{References}

Adam, C., Klissouras, V., Ravazzolo, M., Renson, R., \& Tuxworth, W. (1988). EUROFIT: European test of physical fitness. Rome: Council of Europe, Committee for the development of sport, 10-70.

Al-Hariri, M. T., Elkilany, A. M., \& Alkahtani, S. A. (2018). Effects of potentially modifiable risk factors on the health of adults in the Eastern Province of KSA. $J$ Taibah Univ Med Sci., 13, 16-21. https://doi.org/10.1016/j.jtumed.2017.08.002

Alkahtani, S., Elkilany, A., \& Alhariri, M. (2015). Association between sedentary and physical activity patterns and risk factors of metabolic syndrome in Saudi men: a cross-sectional study. BMC public health, 15(1), 1234. https://doi.org/10.1186/s12889-015-2578-4

Armstrong, N. (2012). Young people are fit and active-Fact or fiction? J SPORT HEALTH SCI, 1, 131-140. https://doi.org/10.1016/j.jshs.2012.05.003

Berisha, M., \& Cilli, M. (2017). Comparison of Eurofit Test Results of 11-17-Year-Old Male and Female Students in Kosovo. European Scientific Journal, ESJ, 13(31). http://doi.org/10.19044/esj.2017.v13n31p138

Erikoğlu, Ö., Güzel, N. A., Pense, M., \& Örer, G. E. (2015). Comparison of Physical Fitness Parameters with EUROFIT Test Battery of Male Adolescent Soccer Players and Sedentary Counterparts. Int JSCS, 3, 43-52.

Lovecchio, N., Casolo, F., Invernizzi, P., \& Eid, L. (2012). Strength in young Italian students: results from Eurofit test and comparison among European data. Polish Journal of Sport and Tourism, 19, 13-15. https://doi.org/10.2478/v10197-012-0002-5

Mabry, R., Reeves, M. M., Eakin, E. G., \&Owen, N. (2010). Evidence of physical activity participation among men and women in the countries of the Gulf Cooperation Council: a review. Obesity reviews, 11, 457-464. http//doi.org/10.1111/j.1467-789X.2009.00655.x

Musaiger, A. (2012). The Food Dome; dietary guidelines for Arab countries. Nutricion hospitalaria, 27. https://doi.org/10.1590/S0212-16112012000100012

Ng, S. W., Zaghloul, S., Ali, H., Harrison, G., Yeatts, K., El Sadig, M., \& Popkin, B. M. (2011). Nutrition transition in the United Arab Emirates. Eur J Clin Nutr, 65(12), 1328. https://doi.org/10.1038/ejen.2011.135

Ortega, F. B., Silventoinen, K., Tynelius, P., \& Rasmussen, F. (2012). Muscular strength in male adolescents and premature death: cohort study of one million participants. Bmj, 345, e7279. https://doi.org/10.1136/bmj.e7279

Reilly, J. J., \& Kelly, J. (2011). Long-term impact of overweight and obesity in childhood and adolescence on morbidity and premature mortality in adulthood: systematic review. International journal of obesity, 35(7), 891. https://doi.org/10.1038/ijo.2010.222

Ruiz, J. R., Castro-Piñero, J., Artero, E. G., Ortega, F. B., Sjöström, M., Suni, J., \& Castillo, M. J. (2009). Predictive validity of health-related fitness in youth: a systematic review. $\mathrm{Br} J$ Sports Med. https//doi.org/10.1136/bjsm.2008.056499

Vancampfort, D., Sienaert, P., Wyckaert, S., De Hert, M., Stubbs, B., Rosenbaum, S., ... \& Probst, M. (2015). Test-retest reliability, feasibility and clinical correlates of the Eurofit test battery in people with bipolar disorder. Psychiatry research, 228(3), 620-625. http//doi.org/10.1016/j.psychres.2015.05.042

Washi, S. A., \& Ageib, M. B. (2010). Poor diet quality and food habits are related to impaired nutritional status in 13-to 18-year-old adolescents in Jeddah. Nut Res, 30(8), 527-534. https://doi.org/10.1016/j.nutres.2010.07.002

World Health Organization [WHO]. (2004). Global strategy on diet, physical activity and health.

Yurdalan, S. U., Kondu, S., \& Malkoç, M. (2007). Assessment of health-related fitness in the patients with end-stage renal disease on hemodialysis: using Eurofit Test Battery. Renal failure, 29(8), 955-960. https//doi.org/10.1080/08860220701641330

\section{Copyrights}

Copyright for this article is retained by the author(s), with first publication rights granted to the journal.

This is an open-access article distributed under the terms and conditions of the Creative Commons Attribution license (http://creativecommons.org/licenses/by/4.0/). 